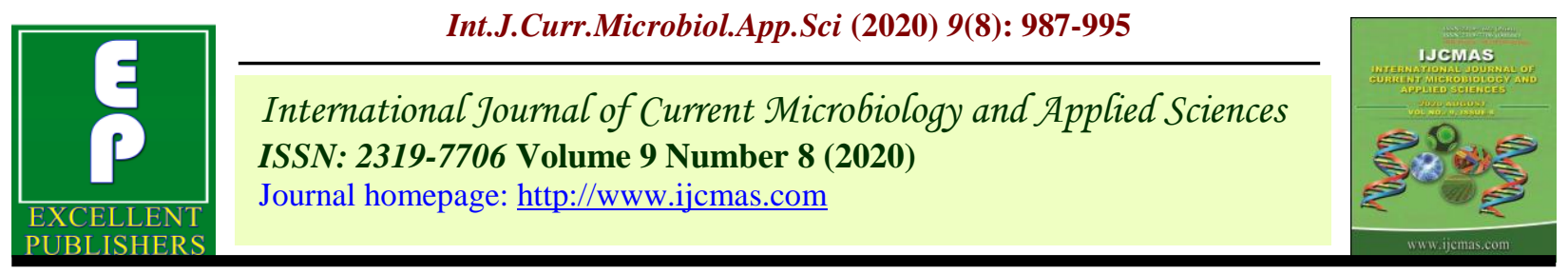

Original Research Article

https://doi.org/10.20546/ijcmas.2020.908.107

\title{
Effect of Integrated Fertilization on Qualitative and Quantitative Traits of Radish (Raphanus sativus L.)
}

\author{
Ankita Mishra*, Sudha Singh and Anurag Greene \\ Department of Horticulture, BFIT Group of Institutions, Suddhowala, Dehradun, \\ Uttrakhand, India \\ *Corresponding author
}

\begin{tabular}{|l|}
\hline K e y w o r d s \\
$\begin{array}{l}\text { INM, Organic, } \\
\text { Inorganic }\end{array}$ \\
\hline Article Info \\
\hline $\begin{array}{l}\text { Accepted: } \\
\text { 10 July 2020 } \\
\text { Available Online: } \\
\text { 10 August } 2020\end{array}$ \\
\hline
\end{tabular}

\section{A B S T R A C T}

An investigation was conducted during the Rabi season 2018-19 at main Experimental Station, Department of Horticulture, BFIT Group of Institutions, Suddhowala, Dehradun, Uttrakhand (U.K), during the Rabi season of 2018-19. The experiment was laid out in Randomized block design (RBD) thrice including ten treatments viz., $\mathrm{T}_{1}$ ControlRecommended dose of fertilizer $\left(100 \mathrm{Kg} \mathrm{N}, 80 \mathrm{Kg} \mathrm{P}_{2} \mathrm{O}_{5}\right.$ and $\left.50 \mathrm{Kg} \mathrm{K}_{2} \mathrm{O} / \mathrm{ha}\right), \mathrm{T}_{2}(100 \% \mathrm{~N}$ through FYM), $\mathrm{T}_{3}\left(100 \% \mathrm{~N}\right.$ through Vermicompost), $\mathrm{T}_{4}(100 \% \mathrm{~N}$ through Poultry manure), $\mathrm{T}_{5}(75 \% \mathrm{NPK}+25 \% \mathrm{~N}$ through $\mathrm{FYM}), \mathrm{T}_{6}(75 \% \mathrm{NPK}+25 \% \mathrm{~N}$ through Vermicompost), $\mathrm{T}_{7}\left(75 \% \mathrm{NPK}+25 \% \mathrm{~N}\right.$ through Poultry manure), $\mathrm{T}_{8}(50 \% \mathrm{NPK}+50 \%$ $\mathrm{N}$ through FYM $), \mathrm{T}_{9}(50 \% \mathrm{NPK}+50 \% \mathrm{~N}$ through Vermicompost $)$ and $\mathrm{T}_{10}(50 \% \mathrm{NPK}+$ $50 \% \mathrm{~N}$ through Poultry manure). The significantly highest plant height $(35.23 \mathrm{~cm})$ at 45 days was observed in $\mathrm{T}_{6}$. The maximum number of leaves per plant (13.50) was recorded in $\left(\mathrm{T}_{6}\right)$. The maximum leaf length (29.97) and the highest shoot weight (91.50) were found in treatment $\left(\mathrm{T}_{6}\right)$. Highest root yield (392.83 q/ha) and highest T.S.S. (5.00) was found in treatment $\mathrm{T}_{6}(75 \% \mathrm{NPK}+25 \% \mathrm{~N}$ through vermicompost) which was significantly superior over all other treatments under study.

\section{Introduction}

Radish (Raphanus sativus L.) is a popular root vegetable of Brassicaceae family with chromosome number $2 \mathrm{n}=18$ commonly called as Mooli, grown all over the world. It most likely originated in the area between the Mediterranean and the Caspian Sea. Radish is grown for its young tender tuberous root which is consumed either cooked or raw. Usually, people eat radishes raw, as a crunchy vegetable, mainly in the salad, while it also appears in many European dishes and young leaves and pods are also cooked as vegetables. Some people, at least in the Middle East prefer to drink its juice in pursuit of health benefits. In homeopathy, it is used for headache, sleeplessness and chronic diarrhoea. It is also useful in urinary complaints scale. In addition, it contains various water-soluble vitamins ie- $\mathrm{B}_{1}, \mathrm{~B}_{2}, \mathrm{~B}_{3}$, $\mathrm{B}_{5}, \mathrm{~B}_{6}$, and $\mathrm{B}_{9}$. It is a good source of vitamins $\mathrm{C}$ (Ascorbic acid) $15-40 \mathrm{mg}$ per $100 \mathrm{~g}$ and minerals like calcium, potassium, and 
phosphorus and piles. The characteristics; pungent flavour of radish is due to the presence of volatile isothiocyanates. Being a short-duration and quick-growing crop, the growth of the roots should be rapid and uninterrupted. Hence, for the production of good quality radish, optimum fertilization through organic, inorganic and biofertilizers are essential. Radishes are quick-germinating, fast-growing vegetables that can give new gardeners a sense of accomplishment withlout taxing their patience. Because radish is a root vegetable, fertilizer needs are for nutrients that promote strong stem and root growth without overly large leaves. Very few soils supply nutrients in the quantities needed for optimum growth of vegetables. Supplementation with organic or inorganic fertilizers allows the gardener to fine tune the amounts to the needs of each type of plant. Leafy vegetables, for instance, need more nitrogen than those grown for their fruits or roots. Both organic and inorganic fertilization have been recommended for radish crop.. Fertilizers use has caused leaching and run-off of nutrients, especially nitrogen $(\mathrm{N})$ and phosphorus $(\mathrm{P})$, leading to environmental degradation. Therefore, there must be a balance between optimal nutrient use efficiency and optimal crop productivity. Amongst the several indicators of soil degradation, over mining of nutrients is considered to be the major concern particularly under vegetable based cropping systems, which have high irrigation requirement. This is happening so because nutrient removal by crops from soil has far exceeded their replenishment through fertilizers and manures causing the negative balance of nutrients in the soil.

The potential way to decrease negative environmental impacts resulting from the inefficient use of chemical fertilizers is to follow the integrated use of organic manures, mineral fertilizers and inoculation with biofertilizers plant growth promoting rhizobacteria (PGPR). This will in turn help to meet out the nutrient requirement of the crops as well as maintaining sustainability in terms of productivity and soil fertility.

\section{Materials and Methods}

The current investigation pertaining to the studies on the "Effect of Integrated fertilization on qualitative and quantitative traits of Radish (Raphanus sativus L.) was conducting during 2018-2019 at main experimental station, Department of Horticulture, BFIT Group of Institutions, Suddhowala Dehradun, Uttrakhand. The experiment will be conducted in Randomized Block Design (RBD) with three replications and 10 treatment i.e., $\mathrm{T}_{1}$ ControlRecommended dose of fertilizer $(100 \mathrm{Kg} \mathrm{N}$, $80 \mathrm{Kg} \mathrm{P}_{2} \mathrm{O}_{5}$ and $\left.50 \mathrm{Kg} \mathrm{K}_{2} \mathrm{O} / \mathrm{ha}\right), \mathrm{T}_{2}(100 \% \mathrm{~N}$ through FYM $), \quad \mathrm{T}_{3} \quad(100 \% \quad \mathrm{~N}$ through Vermicompost), $\mathrm{T}_{4}(100 \% \mathrm{~N}$ through Poultry manure), $\mathrm{T}_{5}(75 \% \mathrm{NPK}+25 \% \mathrm{~N}$ through FYM $), \mathrm{T}_{6}(75 \% \mathrm{NPK}+25 \% \mathrm{~N}$ through Vermicompost), $\mathrm{T}_{7}(75 \% \mathrm{NPK}+25 \% \mathrm{~N}$ through Poultry manure), $\mathrm{T}_{8}(50 \% \mathrm{NPK}+$ $50 \% \mathrm{~N}$ through FYM), $\mathrm{T}_{9}(50 \% \mathrm{NPK}+50 \%$ $\mathrm{N}$ through Vermicompost), $\mathrm{T}_{10}(50 \% \mathrm{NPK}+$ $50 \% \mathrm{~N}$ through Poultry manure). The variance of the measure of the variability and is defined a the average of the square deviation from the mean. The analysis of variance was carried out as per methods suggested by Panse and Sukhatme (1989).

\section{Results and Discussion}

A variety of treatments showed considerable changes in growth parameters viz., plant height, number of leaves per plant, length of leaves, fresh weight of shoots and dry weight of shoots. The height of the plant and the number of leaves per plant are recorded at different intervals. 


\section{Growth parameters}

Maximum plant height of $14.43,32.37$ and $35.37 \mathrm{~cm}$ was recorded highest in treatment $\mathrm{T}_{6} \quad(75 \% \mathrm{NPK}+25 \% \mathrm{~N}$ through vermicompost) at 30,45 DAS and at harvesting stage (Table 1). Number of leaves per plant was affected significantly with different treatments at all stages of growth under study. Treatment $\mathrm{T}_{6}(75 \% \mathrm{NPK}+25 \%$ $\mathrm{N}$ through vermicompost) recorded significantly higher number of leaves per plant i.e. $6.53,11.03$ and 13.50 at 30 and 45 DAS and at harvesting stage, respectively as compared to other treatments It was followed by $\mathrm{T}_{7}$ (5.87, 10.07 and 12.47) (Table 2). Maximum length of leaves recorded at 30, 45 DAS and at harvesting stage was 11.33, 27.03 and $29.97 \mathrm{~cm}$, respectively under the treatment $\mathrm{T}_{6}(75 \% \mathrm{NPK}+25 \% \mathrm{~N}$ through vermicompost) which was superior than other treatments (Table 3). Maximum fresh weight of shoot i.e. 12.73, 83.67 and $91.50 \mathrm{~g}$ was recorded at 30, 45 DAS and at harvesting stage, respectively under the treatment $\mathrm{T}_{6}(75$ $\% \mathrm{NPK}+25 \% \mathrm{~N}$ through vermicompost) (Table 4). Treatments had exerted significant influence on dry weight of shoot in radish at all the stages under study. Treatment $\mathrm{T}_{6}(75 \%$ $\mathrm{NPK}+25 \% \mathrm{~N}$ through vermicompost) recorded maximum dry weight i.e. 1.24, 8.21 and $9.20 \mathrm{~g}$ at $30,45 \mathrm{DAS}$ and at harvesting stage, respect (Table 5). Related results have been described by Kumar et al., (2014), Bhattarai and Maharjan (2013), Uddain et al., (2010).

Table.1 Effect of organic manures, inorganic fertilizers and their combinations on Plant height of radish

\begin{tabular}{|c|c|c|c|c|}
\hline Symbol & Treatments & $\begin{array}{l}\text { Plant height } \\
\text { (cm) } \\
\text { 30 DAS }\end{array}$ & $\begin{array}{l}\text { Plant height } \\
\text { (cm) } \\
45 \text { DAS }\end{array}$ & $\begin{array}{l}\text { Plant height }(\mathbf{c m}) \\
\text { At harvesting } \\
\text { (DAS) }\end{array}$ \\
\hline $\mathbf{T}_{1}$ & $\begin{array}{l}\text { Control-Recommended dose of } \\
\text { fertilizer }\left(100 \mathrm{Kg} \mathrm{N}, 80 \mathrm{Kg} \mathrm{P}_{2} \mathrm{O}_{5}\right. \\
\left.\text { and } 50 \mathrm{Kg} \mathrm{K}_{2} \mathrm{O} / \mathrm{ha}\right)\end{array}$ & 10.47 & 25.73 & 27.73 \\
\hline $\mathbf{T}_{2}$ & $100 \% \mathrm{~N}$ through FYM & 9.13 & 22.83 & 25.90 \\
\hline $\mathbf{T}_{3}$ & $100 \% \mathrm{~N}$ through Vermicompost & 10.20 & 24.97 & 26.93 \\
\hline $\mathbf{T}_{4}$ & $100 \% \mathrm{~N}$ through Poultry manure & 9.80 & 23.77 & 26.07 \\
\hline $\mathbf{T}_{5}$ & $75 \% \mathrm{NPK}+25 \% \mathrm{~N}$ through FYM & 11.90 & 27.30 & 29.67 \\
\hline $\mathbf{T}_{6}$ & $\begin{array}{l}75 \% \mathrm{NPK}+25 \% \mathrm{~N} \text { through } \\
\text { Vermicompost }\end{array}$ & 14.43 & 32.37 & 35.23 \\
\hline $\mathbf{T}_{7}$ & $\begin{array}{l}75 \% \mathrm{NPK}+25 \% \mathrm{~N} \text { through } \\
\text { Poultry manure }\end{array}$ & 13.07 & 29.53 & 32.53 \\
\hline $\mathbf{T}_{8}$ & $50 \% \mathrm{NPK}+50 \% \mathrm{~N}$ through FYM & 10.63 & 25.83 & 27.90 \\
\hline $\mathbf{T}_{9}$ & $\begin{array}{l}50 \% \mathrm{NPK}+50 \% \mathrm{~N} \text { through } \\
\text { Vermicompost }\end{array}$ & 11.63 & 26.97 & 29.10 \\
\hline $\mathbf{T}_{10}$ & $\begin{array}{l}50 \% \mathrm{NPK}+50 \% \mathrm{~N} \text { through } \\
\text { Poultry manure }\end{array}$ & 10.97 & 26.70 & 28.20 \\
\hline \multicolumn{2}{|r|}{ 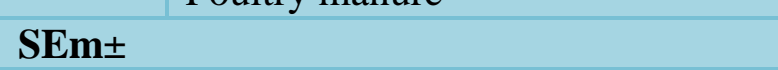 } & 0.45 & 0.94 & 0.89 \\
\hline \multicolumn{2}{|c|}{ CD at $5 \%$} & 1.34 & 2.81 & 2.65 \\
\hline
\end{tabular}


Table.2 Effect of organic manure, inorganic fertilizers and there combination on number of leaves per plant

\begin{tabular}{|c|c|c|c|c|}
\hline Symbol & Treatments & $\begin{array}{c}\text { Number of } \\
\text { leaves per } \\
\text { plant } \\
\text { 30 DAS }\end{array}$ & $\begin{array}{c}\text { Number of } \\
\text { leaves per plant } \\
45 \text { DAS }\end{array}$ & $\begin{array}{l}\text { Number of } \\
\text { leaves per } \\
\text { plant } \\
\text { At harvesting } \\
\text { (DAS) }\end{array}$ \\
\hline $\mathbf{T}_{1}$ & $\begin{array}{l}\text { Control-Recommended dose of fertilizer ( } 100 \\
\left.\mathrm{Kg} \mathrm{N}, 80 \mathrm{Kg} \mathrm{P}_{2} \mathrm{O}_{5} \text { and } 50 \mathrm{Kg} \mathrm{K}_{2} \mathrm{O} / \mathrm{ha}\right)\end{array}$ & 4.87 & 8.63 & 11.13 \\
\hline $\mathbf{T}_{2}$ & $100 \% \mathrm{~N}$ through FYM & 4.60 & 7.87 & 10.83 \\
\hline T3 & $100 \% \mathrm{~N}$ through Vermicompost & 4.77 & 8.23 & 10.93 \\
\hline $\mathbf{T}_{4}$ & $100 \% \mathrm{~N}$ through Poultry manure & 4.63 & 8.10 & 10.97 \\
\hline $\mathbf{T}_{5}$ & $75 \% \mathrm{NPK}+25 \% \mathrm{~N}$ through FYM & 5.27 & 9.03 & 11.43 \\
\hline $\mathbf{T}_{6}$ & $75 \% \mathrm{NPK}+25 \% \mathrm{~N}$ through Vermicompost & 6.53 & 11.03 & 13.50 \\
\hline $\mathbf{T}_{7}$ & $75 \% \mathrm{NPK}+25 \% \mathrm{~N}$ through Poultry manure & 5.87 & 10.07 & 12.47 \\
\hline $\mathbf{T}_{8}$ & $50 \% \mathrm{NPK}+50 \% \mathrm{~N}$ through FYM & 4.97 & 8.70 & 11.23 \\
\hline $\mathbf{T}_{9}$ & $50 \% \mathrm{NPK}+50 \% \mathrm{~N}$ through Vermicompost & 5.20 & 8.80 & 11.37 \\
\hline $\mathbf{T}_{10}$ & $50 \% \mathrm{NPK}+50 \% \mathrm{~N}$ through Poultry manure & 5.10 & 8.77 & 11.30 \\
\hline \multicolumn{2}{|r|}{ 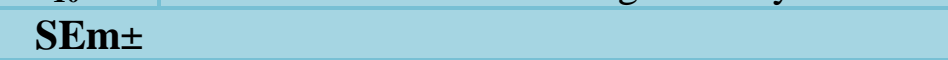 } & 0.18 & 0.32 & 0.34 \\
\hline \multicolumn{2}{|c|}{ CD at $5 \%$} & 0.54 & 0.96 & 1.02 \\
\hline
\end{tabular}

Table.3 Effect of organic manures, inorganic fertilizers and their combinations on length of leaves $(\mathrm{cm})$ of radish

\begin{tabular}{|c|c|c|c|c|}
\hline Symbol & Treatments & $\begin{array}{l}\text { Length of leaves } \\
\text { (cm) } 30 \text { DAS }\end{array}$ & $\begin{array}{c}\text { Length of leaves }(\mathrm{cm}) \\
45 \text { DAS }\end{array}$ & $\begin{array}{c}\text { Length of leaves (cm) } \\
\text { At harvesting (DAS) }\end{array}$ \\
\hline $\mathbf{T}_{1}$ & $\begin{array}{l}\text { Control-Recommended dose of } \\
\text { fertilizer }\left(100 \mathrm{Kg} \mathrm{N}, 80 \mathrm{Kg} \mathrm{P}_{2} \mathrm{O}_{5}\right. \\
\left.\text { and } 50 \mathrm{Kg} \mathrm{K}_{2} \mathrm{O} / \mathrm{ha}\right)\end{array}$ & 8.63 & 20.77 & 24.27 \\
\hline $\mathbf{T}_{2}$ & $100 \% \mathrm{~N}$ through FYM & 6.83 & 18.43 & 21.83 \\
\hline T3 & $100 \% \mathrm{~N}$ through Vermicompost & 7.80 & 20.03 & 23.19 \\
\hline $\mathbf{T}_{4}$ & $100 \% \mathrm{~N}$ through Poultry manure & 7.67 & 19.69 & 23.01 \\
\hline $\mathbf{T}_{5}$ & $75 \% \mathrm{NPK}+25 \% \mathrm{~N}$ through FYM & 9.10 & 22.25 & 25.21 \\
\hline $\mathbf{T}_{6}$ & $\begin{array}{l}75 \% \mathrm{NPK}+25 \% \mathrm{~N} \text { through } \\
\text { Vermicompost }\end{array}$ & 11.33 & 27.03 & 29.97 \\
\hline $\mathbf{T}_{7}$ & $\begin{array}{l}75 \% \mathrm{NPK}+25 \% \mathrm{~N} \text { through } \\
\text { Poultry manure }\end{array}$ & 10.17 & 24.33 & 27.49 \\
\hline $\mathbf{T}_{8}$ & $50 \% \mathrm{NPK}+50 \% \mathrm{~N}$ through FYM & 8.70 & 21.70 & 24.45 \\
\hline $\mathbf{T}_{9}$ & $\begin{array}{l}50 \% \mathrm{NPK}+50 \% \mathrm{~N} \text { through } \\
\text { Vermicompost }\end{array}$ & 9.07 & 22.01 & 25.20 \\
\hline $\mathbf{T}_{10}$ & $\begin{array}{l}50 \% \mathrm{NPK}+50 \% \mathrm{~N} \text { through Poultry } \\
\text { manure }\end{array}$ & 8.80 & 21.95 & 24.56 \\
\hline \multicolumn{2}{|r|}{ 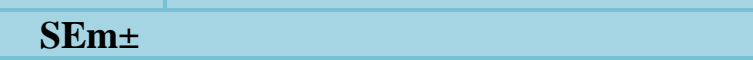 } & 0.37 & 0.90 & 0.77 \\
\hline \multicolumn{2}{|c|}{ CD at $5 \%$} & 1.12 & 2.69 & 2.30 \\
\hline
\end{tabular}


Table.4 Effect of organic manures, inorganic fertilizers and their combinations on fresh weight of shoot $(\mathrm{g})$ of radish

\begin{tabular}{|c|c|c|c|c|}
\hline Symbol & Treatments & $\begin{array}{c}\text { Fresh } \\
\text { weight of } \\
\text { shoot (g) } \\
\text { 30 DAS }\end{array}$ & $\begin{array}{l}\text { Fresh weight of } \\
\text { shoot (g) } \\
\text { 45 DAS }\end{array}$ & $\begin{array}{c}\text { Fresh weight of } \\
\text { shoot (g) } \\
\text { At harvesting } \\
\text { (DAS) }\end{array}$ \\
\hline $\mathbf{T}_{1}$ & $\begin{array}{l}\text { Control-Recommended dose of fertilizer (100 } \\
\left.\mathrm{Kg} \mathrm{N}, 80 \mathrm{Kg} \mathrm{P}_{2} \mathrm{O}_{5} \text { and } 50 \mathrm{Kg} \mathrm{K}_{2} \mathrm{O} / \mathrm{ha}\right)\end{array}$ & 9.03 & 63.50 & 77.03 \\
\hline $\mathbf{T}_{2}$ & $100 \% \mathrm{~N}$ through FYM & 8.03 & 59.67 & 74.07 \\
\hline T3 & $100 \% \mathrm{~N}$ through Vermicompost & 8.63 & 61.07 & 76.67 \\
\hline $\mathbf{T}_{4}$ & $100 \% \mathrm{~N}$ through Poultry manure & 8.57 & 60.33 & 75.90 \\
\hline $\mathbf{T}_{5}$ & $75 \%$ NPK $+25 \% \mathrm{~N}$ through FYM & 10.43 & 71.33 & 80.33 \\
\hline $\mathbf{T}_{6}$ & $75 \% \mathrm{NPK}+25 \% \mathrm{~N}$ through Vermicompost & 12.73 & 83.67 & 91.50 \\
\hline $\mathbf{T}_{7}$ & $75 \% \mathrm{NPK}+25 \% \mathrm{~N}$ through Poultry manure & 11.50 & 77.37 & 85.87 \\
\hline $\mathbf{T}_{8}$ & $50 \% \mathrm{NPK}+50 \% \mathrm{~N}$ through FYM & 9.40 & 65.17 & 77.18 \\
\hline $\mathbf{T}_{\mathbf{9}}$ & $50 \% \mathrm{NPK}+50 \% \mathrm{~N}$ through Vermicompost & 10.37 & 70.83 & 80.30 \\
\hline $\mathbf{T}_{10}$ & $50 \% \mathrm{NPK}+50 \% \mathrm{~N}$ through Poultry manure & 9.67 & 70.00 & 79.33 \\
\hline \multicolumn{2}{|l|}{ SEm \pm} & 0.41 & 2.12 & 1.85 \\
\hline \multicolumn{2}{|c|}{ CD at $5 \%$} & 1.22 & 6.31 & 5.50 \\
\hline
\end{tabular}

Table5 Effect of organic manures, inorganic fertilizers and their combinations on dry weight of shoot (g) of radish

\begin{tabular}{|c|c|c|c|c|}
\hline Symbol & Treatments & $\begin{array}{l}\text { Dry weight of } \\
\text { shoot (g) } \\
\text { 30 DAS }\end{array}$ & $\begin{array}{l}\text { Dry weight of } \\
\text { shoot (g) } \\
45 \text { DAS }\end{array}$ & $\begin{array}{l}\text { Dry weight of } \\
\text { shoot }(\mathrm{g}) \\
\text { At harvesting } \\
\text { (DAS) }\end{array}$ \\
\hline $\mathbf{T}_{1}$ & $\begin{array}{l}\text { Control-Recommended dose of fertilizer ( } 100 \\
\mathrm{Kg} \mathrm{N}, 80 \mathrm{Kg} \mathrm{P}_{2} \mathrm{O}_{5} \text { and } 50 \mathrm{Kg} \mathrm{K}_{2} \mathrm{O} / \mathrm{ha} \text { ) }\end{array}$ & 0.90 & 6.28 & 7.64 \\
\hline $\mathbf{T}_{2}$ & $100 \% \mathrm{~N}$ through FYM & 0.80 & 5.57 & 7.27 \\
\hline T3 & $100 \% \mathrm{~N}$ through Vermicompost & 0.85 & 5.91 & 7.43 \\
\hline $\mathbf{T}_{4}$ & $100 \% \mathrm{~N}$ through Poultry manure & 0.86 & 5.87 & 7.41 \\
\hline $\mathbf{T}_{5}$ & $75 \% \mathrm{NPK}+25 \% \mathrm{~N}$ through FYM & 1.00 & 6.80 & 7.87 \\
\hline $\mathbf{T}_{6}$ & $75 \%$ NPK $+25 \% \mathrm{~N}$ through Vermicompost & 1.24 & 8.21 & 9.20 \\
\hline $\mathbf{T}_{7}$ & $75 \% \mathrm{NPK}+25 \% \mathrm{~N}$ through Poultry manure & 1.11 & 7.50 & 8.47 \\
\hline $\mathbf{T}_{8}$ & $50 \% \mathrm{NPK}+50 \% \mathrm{~N}$ through FYM & 0.92 & 6.51 & 7.67 \\
\hline $\mathbf{T}_{9}$ & $50 \% \mathrm{NPK}+50 \% \mathrm{~N}$ through Vermicompost & 1.00 & 6.61 & 7.77 \\
\hline $\mathbf{T}_{10}$ & $50 \% \mathrm{NPK}+50 \% \mathrm{~N}$ through Poultry manure & 0.93 & 6.73 & 7.73 \\
\hline \multicolumn{2}{|r|}{ 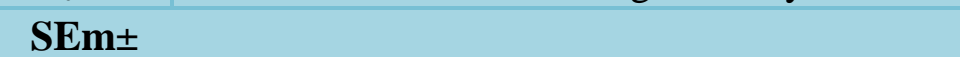 } & 0.04 & 0.24 & 0.24 \\
\hline \multicolumn{2}{|c|}{ CD at $5 \%$} & 0.12 & 0.71 & 0.72 \\
\hline
\end{tabular}


Table.6 Effect of organic manures, inorganic fertilizers and their combinations on length of root $(\mathrm{cm})$ and diameter of root $(\mathrm{cm})$ of radish

\begin{tabular}{|l|l|c|c|}
\hline Symbol & Treatments & $\begin{array}{c}\text { Length of root } \\
(\mathbf{c m})\end{array}$ & $\begin{array}{c}\text { Diameter of root } \\
\text { (cm) }\end{array}$ \\
\hline $\mathbf{T}_{\mathbf{1}}$ & $\begin{array}{l}\text { Control-Recommended dose of fertilizer }(100 \\
\left.\mathrm{Kg} \mathrm{N}, 80 \mathrm{Kg} \mathrm{P}_{2} \mathrm{O}_{5} \text { and } 50 \mathrm{Kg} \mathrm{K}{ }_{2} \mathrm{O} / \mathrm{ha}\right)\end{array}$ & 17.07 & 2.88 \\
\hline $\mathbf{T}_{\mathbf{2}}$ & $100 \% \mathrm{~N}$ through FYM & 15.80 & 2.74 \\
\hline $\mathbf{T 3}_{3}$ & $100 \% \mathrm{~N}$ through Vermicompost & 16.97 & 2.81 \\
\hline $\mathbf{T}_{\mathbf{4}}$ & $100 \% \mathrm{~N}$ through Poultry manure & 16.89 & 2.80 \\
\hline $\mathbf{T}_{\mathbf{5}}$ & $75 \% \mathrm{NPK}+25 \% \mathrm{~N}$ through FYM & 17.83 & 3.11 \\
\hline $\mathbf{T}_{\mathbf{6}}$ & $75 \% \mathrm{NPK}+25 \% \mathrm{~N}$ through Vermicompost & 19.97 & 3.84 \\
\hline $\mathbf{T}_{\mathbf{7}}$ & $75 \% \mathrm{NPK}+25 \%$ N through Poultry manure & 18.30 & 3.48 \\
\hline $\mathbf{T}_{\mathbf{8}}$ & $50 \% \mathrm{NPK}+50 \% \mathrm{~N}$ through FYM & 17.30 & 2.96 \\
\hline $\mathbf{T}_{\mathbf{9}}$ & $50 \% \mathrm{NPK}+50 \% \mathrm{~N}$ through Vermicompost & 17.83 & 3.03 \\
\hline $\mathbf{T}_{\mathbf{1 0}}$ & $50 \% \mathrm{NPK}+50 \% \mathrm{~N}$ through Poultry manure & 17.67 & 2.99 \\
\hline SEm \pm & & $\mathbf{0 . 5 5}$ & $\mathbf{0 . 1 2}$ \\
\hline CD at 5\% & $\mathbf{1 . 6 5}$ & $\mathbf{0 . 3 6}$ \\
\hline
\end{tabular}

Table.7 Effect of organic manures, inorganic fertilizers and their combinations on weight of root (g) and root yield (q/ha) of radish

\begin{tabular}{|c|c|c|c|}
\hline Symbol & Treatments & $\begin{array}{l}\text { Average weight of } \\
\operatorname{root}(\mathrm{g})\end{array}$ & $\begin{array}{l}\text { Root yield } \\
\text { (q/ha) }\end{array}$ \\
\hline $\mathbf{T}_{1}$ & $\begin{array}{l}\text { Control-Recommended dose of fertilizer ( } 100 \\
\left.\mathrm{Kg} \mathrm{N}, 80 \mathrm{Kg} \mathrm{P}_{2} \mathrm{O}_{5} \text { and } 50 \mathrm{Kg} \mathrm{K}_{2} \mathrm{O} / \mathrm{ha}\right)\end{array}$ & 91.20 & 303.97 \\
\hline $\mathbf{T}_{2}$ & $100 \% \mathrm{~N}$ through FYM & 81.48 & 271.57 \\
\hline T3 & $100 \% \mathrm{~N}$ through Vermicompost & 90.03 & 300.08 \\
\hline $\mathbf{T}_{4}$ & $100 \% \mathrm{~N}$ through Poultry manure & 83.10 & 276.97 \\
\hline $\mathbf{T}_{5}$ & $75 \% \mathrm{NPK}+25 \% \mathrm{~N}$ through FYM & 101.07 & 336.88 \\
\hline $\mathbf{T}_{6}$ & $75 \% \mathrm{NPK}+25 \% \mathrm{~N}$ through Vermicompost & 117.86 & 392.83 \\
\hline $\mathbf{T}_{7}$ & $75 \% \mathrm{NPK}+25 \% \mathrm{~N}$ through Poultry manure & 101.21 & 337.34 \\
\hline $\mathbf{T}_{8}$ & $50 \% \mathrm{NPK}+50 \% \mathrm{~N}$ through FYM & 93.53 & 311.75 \\
\hline $\mathbf{T}_{\mathbf{9}}$ & $50 \% \mathrm{NPK}+50 \% \mathrm{~N}$ through Vermicompost & 98.80 & 329.30 \\
\hline $\mathbf{T}_{10}$ & $50 \% \mathrm{NPK}+50 \% \mathrm{~N}$ through Poultry manure & 93.58 & 311.89 \\
\hline \multicolumn{2}{|c|}{ SEm \pm} & 3.33 & 11.11 \\
\hline \multicolumn{2}{|c|}{ CD at $5 \%$} & 9.90 & 33.01 \\
\hline
\end{tabular}


Table.8 Effect of organic manures, inorganic fertilizers and their combinations on day to harvest and harvest index

\begin{tabular}{|c|c|c|c|}
\hline Symbol & Treatments & Day to harvest & Harvest index $(\%)$ \\
\hline $\mathbf{T}_{1}$ & $\begin{array}{l}\text { Control-Recommended dose of fertilizer ( } 100 \mathrm{Kg} \\
\mathrm{N}, 80 \mathrm{Kg} \mathrm{P}_{2} \mathrm{O}_{5} \text { and } 50 \mathrm{Kg} \mathrm{K}_{2} \mathrm{O} / \mathrm{ha} \text { ) }\end{array}$ & 59.00 & 55.45 \\
\hline $\mathbf{T}_{2}$ & $100 \% \mathrm{~N}$ through FYM & 60.33 & 52.28 \\
\hline T3 & $100 \% \mathrm{~N}$ through Vermicompost & 59.17 & 53.99 \\
\hline $\mathbf{T}_{4}$ & $100 \% \mathrm{~N}$ through Poultry manure & 59.67 & 52.38 \\
\hline $\mathbf{T}_{5}$ & $75 \%$ NPK $+25 \% \mathrm{~N}$ through FYM & 57.10 & 54.56 \\
\hline $\mathbf{T}_{6}$ & $75 \% \mathrm{NPK}+25 \% \mathrm{~N}$ through Vermicompost & 51.57 & 56.80 \\
\hline $\mathbf{T}_{7}$ & $75 \%$ NPK $+25 \%$ N through Poultry manure & 54.67 & 54.53 \\
\hline $\mathbf{T}_{8}$ & $50 \% \mathrm{NPK}+50 \% \mathrm{~N}$ through FYM & 58.33 & 54.15 \\
\hline $\mathbf{T}_{9}$ & $50 \% \mathrm{NPK}+50 \% \mathrm{~N}$ through Vermicompost & 57.87 & 54.53 \\
\hline $\mathbf{T}_{10}$ & $50 \% \mathrm{NPK}+50 \% \mathrm{~N}$ through Poultry manure & 58.00 & 54.18 \\
\hline \multicolumn{2}{|c|}{$\mathrm{SEm} \pm$} & 1.04 & 0.76 \\
\hline \multicolumn{2}{|c|}{ CD at $5 \%$} & 3.09 & 2.25 \\
\hline
\end{tabular}

Table.9 Effect of organic manures, inorganic fertilizers and their combinations on fiber content, TSS and ascorbic acid

\begin{tabular}{|c|c|c|c|c|}
\hline Symbol & Treatments & $\begin{array}{c}\text { Fiber } \\
\text { Content } \\
(\mathrm{mg} / 100 \mathrm{~g})\end{array}$ & $\begin{array}{c}\text { TSS } \\
\left({ }^{0} \text { Brix }\right)\end{array}$ & $\begin{array}{l}\text { Ascorbic } \\
\text { acid } \\
(\mathrm{mg} / 100 \mathrm{~g})\end{array}$ \\
\hline $\mathbf{T}_{1}$ & $\begin{array}{l}\text { Control-Recommended dose of } \\
\text { fertilizer }\left(100 \mathrm{Kg} \mathrm{N}, 80 \mathrm{Kg} \mathrm{P}_{2} \mathrm{O}_{5} \text { and } 50\right. \\
\left.\mathrm{Kg} \mathrm{K}_{2} \mathrm{O} / \mathrm{ha}\right)\end{array}$ & 549.67 & 4.13 & 32.33 \\
\hline $\mathbf{T}_{2}$ & $100 \% \mathrm{~N}$ through FYM & 505.33 & 3.81 & 27.00 \\
\hline T3 & $100 \% \mathrm{~N}$ through Vermicompost & 544.00 & 4.05 & 31.33 \\
\hline $\mathbf{T}_{4}$ & $100 \% \mathrm{~N}$ through Poultry manure & 516.00 & 4.03 & 30.67 \\
\hline $\mathbf{T}_{5}$ & $75 \%$ NPK $+25 \% \mathrm{~N}$ through FYM & 640.00 & 4.47 & 33.67 \\
\hline $\mathbf{T}_{6}$ & $\begin{array}{l}75 \% \mathrm{NPK}+25 \% \mathrm{~N} \text { through } \\
\text { Vermicompost }\end{array}$ & 763.67 & 5.00 & 37.17 \\
\hline $\mathbf{T}_{7}$ & $\begin{array}{l}75 \% \mathrm{NPK}+25 \% \mathrm{~N} \text { through Poultry } \\
\text { manure }\end{array}$ & 675.33 & 4.55 & 34.33 \\
\hline $\mathbf{T}_{8}$ & $50 \% \mathrm{NPK}+50 \% \mathrm{~N}$ through FYM & 584.67 & 4.15 & 32.33 \\
\hline $\mathbf{T}_{9}$ & $\begin{array}{l}50 \% \mathrm{NPK}+50 \% \mathrm{~N} \text { through } \\
\text { Vermicompost }\end{array}$ & 608.67 & 4.37 & 33.00 \\
\hline $\mathbf{T}_{10}$ & $\begin{array}{l}50 \% \mathrm{NPK}+50 \% \mathrm{~N} \text { through Poultry } \\
\text { manure }\end{array}$ & 596.00 & 4.25 & 32.67 \\
\hline \multicolumn{2}{|l|}{ SEm \pm} & 27.18 & 0.128 & 0.96 \\
\hline \multicolumn{2}{|c|}{ CD at $5 \%$} & 80.75 & 0.380 & 2.87 \\
\hline
\end{tabular}




\section{Yield parameters}

Maximum root length $(19.97 \mathrm{~cm})$ was observed in treatment $\mathrm{T}_{6}(75 \% \mathrm{NPK}+25 \%$ $\mathrm{N}$ through vermicompost), which was followed by $\mathrm{T}_{7}(50 \% \mathrm{NPK}+50 \% \mathrm{~N}$ through poultry manure) with significant difference. Minimum root length $15.80 \mathrm{~cm}$ was observed in treatment $\mathrm{T}_{2}(100 \% \mathrm{~N}$ through FYM) (Table 6). Maximum diameter of root (3.84 $\mathrm{cm})$ was recorded in treatment $\mathrm{T}_{6}(75 \% \mathrm{NPK}$ $+25 \% \mathrm{~N}$ through vermicompost), which was followed by $\mathrm{T}_{7}(50 \% \mathrm{NPK}+50 \% \mathrm{~N}$ through poultry manure) with significant difference (Table 6), where as minimum diameter of root $(2.74 \mathrm{~cm})$ was recorded under the treatment $\mathrm{T}_{2}(100 \% \mathrm{~N}$ through FYM.) (Table 6).

Application of treatment $\mathrm{T}_{6}(75 \% \mathrm{NPK}+$ $25 \% \mathrm{~N}$ through vermicompost) recorded highest harvest index (56.80) which was significantly superior over other treatments. While minimum harvest index (52.28) was observed under the treatment $\mathrm{T}_{2}(100 \% \mathrm{~N}$ through FYM) (Table 8).

Maximum average weight of root $(117.86 \mathrm{~g})$ was noted under the treatment $\mathrm{T}_{6}(75 \%$ NPK $+25 \% \mathrm{~N}$ through vermicompost), while minimum average weight of root $(81.48 \mathrm{~g})$ was observed under the treatment $\mathrm{T}_{2}(100 \% \mathrm{~N}$ through FYM) (Table 7). Highest root yield (392.83 q/ha) was recorded under the treatment $\mathrm{T}_{6}(75 \% \mathrm{NPK}+25 \% \mathrm{~N}$ through vermicompost), where as lowest root yield of (271.57 q/ha) was observed under the treatment $\mathrm{T}_{2}(100 \% \mathrm{~N}$ through FYM) (Table 7). Treatment $\mathrm{T}_{2}(100 \% \mathrm{~N}$ through FYM) had taken maximum days (60.33) to attain marketable root size. Minimum days (51.57) to attain marketable size of root was observed in treatment $\mathrm{T}_{6}(75 \% \mathrm{NPK}+25 \% \mathrm{~N}$ through Vermicompost). Related results have been described by Sunandarani and Malareddy (2007), Kumar et al., (2014), Degwale (2016), Narayan et al., (2014).

\section{Quality parameter}

Highest fiber content $(763.67 \mathrm{mg} / 100 \mathrm{~g}$ edible portion) was observed in treatment $\mathrm{T}_{6}(75 \%$ $\mathrm{NPK}+25 \% \mathrm{~N}$ through vermicompost), which was followed by $\mathrm{T}_{7}(50 \% \mathrm{NPK}+50 \% \mathrm{~N}$ through poultry manure). Minimum fiber content $(505.33 \mathrm{mg} / 100 \mathrm{~g}$ edible portion) was recorded in treatment $\mathrm{T}_{2}(100 \%+\mathrm{N}$ through FYM) (Table 9). Highest total soluble solid content $\left(5^{\circ}\right.$ Brix $)$ in root was determined in case of $\mathrm{T}_{6}(75 \% \mathrm{NPK}+25 \% \mathrm{~N}$ through vermicompost), which was followed by $\mathrm{T}_{7}$ $(50 \% \mathrm{NPK}+50 \% \mathrm{~N}$ through poultry manure).

Minimum total soluble solid content $\left(3.81^{0}\right.$ Brix) was found in treatment $\mathrm{T}_{2}(100 \%$ $\mathrm{N}$ through FYM) (Table 9). Highest Ascorbic acid $(37.17 \mathrm{mg} / 100 \mathrm{~g})$ was determined in treatment $\mathrm{T}_{6}(75 \% \mathrm{NPK}+25 \% \mathrm{~N}$ through vermicompost) which was significantly superior over other treatments. Lowest Ascorbic acid content (27.00mg/100g) treatment $\mathrm{T}_{2}(100 \% \mathrm{~N}$ through FYM) (Table 9). Related results have been described by Sunandarani and Mallareddy (2007), Kumar et al., (2014) and Degwale (2016).

In conclusion based on the present examination, it was summarised that Radish (Raphanus sativus L.) responed well in terms of growth, yield, and quality, by the application of combination of organic manures and inorganic fertilizers. The significantly highest plant height $(35.23 \mathrm{~cm})$ was recorded in treatment $\left(\mathrm{T}_{6}\right)$. The maximum number of leaves per plant (13.50) was recorded in $\left(\mathrm{T}_{6}\right)$. The maximum leaf length (29.97) and the highest fresh weight of shoot weight (91.50) were found in treatment $\left(\mathrm{T}_{6}\right)$. Highest root yield (392.83 q/ha) and highest T.S.S. (5.00) was found in treatment $\mathrm{T}_{6}(75 \%$ $\mathrm{NPK}+25 \% \mathrm{~N}$ through vermicompost) which was significantly superior over all other treatments under study. 


\section{References}

Bhattarai, B. P. and Maharjan, A. (2013). Effect of organic nutrient management on the growth and yield of carrot (Daucus carota L.) and soil fertility status. Nepalese J. Agric.Sci 11:16-25

Degwale, A. (2016) Effect of vermicompost on growth, yield and quality of garlic (Allium sativum L.) In enebse sar midir district, Northwestern Ethopia. J. Natural Sci. Res. 6 (3): 2224- 3186.

Kumar, S.; Maji, S.; Kumar, S. and Singh, H. D. (2014). Efficacy of organic manures on growth and yield of radish (Raphanus sativus L.) cv. Japanese White. Inter. J. Plant Sci. 9(1): 57-60.

Kumar, P.; Meghwal, P. R. and Painuli, D. K. (2014). Effect of organic and inorganic nutrient source on soil health and quality of carrot.Indian J. Hort.71 (2): 222-226.
Narayan, S.; Kanth, R.H.; Narayan, R.; Khan, F.A.; Saxena, A. and Hussain, T. (2014)

Effect of planting dates and integrated nutrient management on productivity and profitability of potato (Solanum tuberosum) in Kashmir valley. Indian J. Agron. 59 (1): 145-150.

Panse, V.G. and Sukhatme, P.V. (1989). Statistical Methods for Agriculture of Workers. $5^{\text {th }}$ Ed. ICAR. New Delhi.

Sunandarani, N. and Mallareddy, K. (2007). Effect of different organic manures and inorganic fertilizers on growth, yield and quality of carrot (Daucus carota L.) Karnataka J. Agric. Sci. 20 (3): 686688.

Uddain, J.; Chowdhury, S. and Rahman, M.J. (2010). Efficacy of different organicmanures on growth and productivity of radish (Raphanus sativus L.) IJAEB. 3 (2): 189-193.

\section{How to cite this article:}

Ankita Mishra, Sudha Singh and Anurag Greene. 2020. Effect of Integrated Fertilization on Qualitative and Quantitative Traits of Radish (Raphanus sativus L.). Int.J.Curr.Microbiol.App.Sci. 9(08): 987-995. doi: https://doi.org/10.20546/ijcmas.2020.908.107 